
2 Research Square
Preprints are preliminary reports that have not undergone peer review.
They should not be considered conclusive, used to inform clinical practice, or referenced by the media as validated information.

\title{
Expression and Clinical Significance of Origin Recognition Complex Family in Breast Cancer
}

\author{
shaohua chen \\ Guangxi Medical University \\ Ziyao Jin \\ Guilin Medical University \\ Linfeng Xin \\ Guilin Medical University \\ Lv LV \\ Guilin Medical University \\ Xuemei Zhang \\ Guilin Medical University \\ Yizhen Gong \\ Guangxi Medical University \\ Jianlun Liu ( $\sim 631228181 @ q q . c o m$ ) \\ Affiliated Tumor Hospital of Guangxi Medical University
}

\section{Research}

Keywords: ORC, breast cancer, prognostic biomarker, nomogram, gene set enrichment analysis, immune infiltrates

Posted Date: September 20th, 2021

DOI: https://doi.org/10.21203/rs.3.rs-900515/v1

License: @) (i) This work is licensed under a Creative Commons Attribution 4.0 International License. Read Full License 


\section{Abstract}

Background

The aim of this study is to investigate the potential clinical and prognostic value, role and driving molecular mechanisms of the origin recognition complex family in breast cancer.

Results

ata from Oncomine, TCGA, GEO and ULCAN showed that ORC1L and ORC6L were highly expressed in breast cancer tissues, while the expression of ORC5L was inconsistent and there was no significant difference in the expression of ORC2L, ORC3L and ORC4L. High expression of ORC1L and ORC6L were mainly Her2 overexpressed subtype, and their expression were negatively correlated with patient age and positively correlated with tumor size, but not with lymph node metastasis, distant metastasis, or tumor stage. Expression of ORC5L was also negatively correlated with age and positively correlated with lymph node metastasis, but not with breast cancer molecular subtype and tumor size. Expression of ORC1L and ORC5L had high diagnostic value, and ORC6L had the highest diagnostic value in breast cancer. ORC6L was an independent poor prognostic factor for overall survival of breast cancer patients. It was involved in cell cycle progression, cell senescence, epigenetic regulation and other biological functions, and may regulate signaling pathways such as NF-KB, TP53 and WNT in breast cancer. We also found that the expression of ORC6L was related to the increased infiltration of Th1/2 cell and Treg cell, and decreased infiltration of Mast cell and NK cell.

Conclusions

ORC1L and ORC6L are highly expressed in breast cancer tissues, of which ORC6L has high diagnostic value and is an independent poor prognostic factor for overall survival of breast cancer patients. ORC6L may be involved in the occurrence and progression of breast cancer by regulating cell cycle progression, promoting the activation of cancer signaling pathways, and influencing tumor immune cells infiltration.

\section{Background}

The latest data show that breast cancer (BC) has surpassed lung cancer to become the most common malignancy in the world. It is the first leading cause of cancer death in women and ranks fifth in cancer-related deaths worldwide ${ }^{1}$. Despite the rapid development of diagnostic methods and treatment strategies in the past decades, BC still faces major challenges, for example, the 5-year survival rate is only $20 \%$, and $5-10 \%$ newly diagnosed $\mathrm{BC}$ patients have distant metastasis ${ }^{2}$. Therefore, exploring early diagnostic and prognostic biomarkers of $\mathrm{BC}$ and discovering the pathogenesis of $\mathrm{BC}$ are critical.

The origin recognition complex (ORC) consists of six subunits, including ORC1L, ORC2L, ORC3L, ORC4L, ORC5L and ORC6L. ORC binds to the origin of DNA replication and regulates the initiation of replication ${ }^{3}$. In recent decades, a large amount of evidence have shown that ORC is involved in other biological processes, such as heterochromatin formation, cytokinesis, viral replication, DNA damage repair, cognitive impairment and radiation inflammatory response $\mathrm{e}^{4-9}$. ORCs are also differentially expressed in a number of tumors, such as gliomas ${ }^{10}$, endometrial cancer ${ }^{11}$, colon cancer $^{12-14}$, gastric cancer ${ }^{15}, 16$, liver cancer ${ }^{17}$, esophageal cancer ${ }^{18}$, and lung cancer ${ }^{19}$. They are implicated in diverse biological processes, including cell proliferation, migration, invasion, tumor stem cell maintenance, and chemotherapy resistance ${ }^{11-13,19-22}$. ORC1 L, ORC5L and ORC6L have prognostic value in gastric cancer, liver cancer and colorectal cancer ${ }^{14-17}$. However, the expression levels and roles of ORCs in BC tumorigenesis remain to be elucidated.

In this current study, we elucidated the expression, clinical application value, and potential molecular mechanism of ORCs in BC, providing a new theoretical basis for the diagnosis and treatment of BC.

\section{Results}

\section{The expression of ORCs in BC}

The ORCs was involved in the malignant progression of multiple tumors. Studies had shown that ORCs were differentially expressed in colorectal cancer, gastric cancer, and lung cancer and were related to biological behaviors such as cell proliferation, migration, and invasion ${ }^{12-16,19}$. Based on the Oncomine database, we also found that at least one of the ORCs was differentially expressed in colorectal cancer, gastric cancer, lung cancer and other tumors. Notably, ORC1L and ORC6L were high expressed and ORC5L was low expressed in BC tissues, which have not been reported before (Fig.1A). Most importantly, the underlying molecular mechanism of ORCs remains indistinct in BC.

We analyzed the prognostic value of ORCs in $7830 \mathrm{BC}$ patients using the KM-plot online database, and found that patients with high ORC5L expression had worse OS [hazard ratio $(H R)=1.22$, 95\% confidence interval $(\mathrm{Cl})=1.01-1.47$, Logrank $\mathrm{P}=0.036$ ]. Similarly, $\mathrm{BC}$ patients with high expression of ORC6L also had worse OS [HR=1.5, $95(\mathrm{Cl})=1.24-1.81$, Logrank P=2.31E-05], i.e., the expression of ORC5L and ORC6L were negatively 
correlated with the OS of BC patients (Fig.1B). While, the expression of ORC1L, ORC2L, ORC3L, and ORC4L was not associated with the OS of BC patients. These results suggest that ORC5L and ORC6L may be potential prognostic factors for BC.

Since genes have tissue-specific expression, we further studied the expression of ORCs in BC combined multiple database. Gene expression data of $1109 \mathrm{BC}$ and 113 normal tissues were obtained from TCGA. Results showed that the mRNA expression of ORC1L, ORC5L, and ORC6L were significantly higher in $\mathrm{BC}$ tissues than those in normal tissues, while the expression of ORC2L, ORC3L, and ORC4L were not significantly different (Fig.2A). Data from GEO datasets (GSE54002, GSE37751) had concordant results (Fig.2B-C). Comprehensive analysis of ONCOMINE, TCGA and GEO, it can be concluded that ORC1L and ORC6L mRNAs were highly expressed in BC tissues, while the expression of ORC5L was inconsistent and there were no significant differences in the expression of ORC2L, ORC3L and ORC4L.

It is well known that proteins are the major contributors of biological function. Then, UALCAN cancer OMICS data were further used to reveal the protein expression of the ORCs in $125 \mathrm{BC}$ and 18 normal tissues. Consistent with the mRNA expression, ORC1L and ORC6L proteins were significantly overexpressed in BC tissues. However, the protein level of ORC5L was low in BC tissues, which was inconsistent at the mRNA level (Fig.2D). Interestingly, the protein levels of ORC2L and ORC3L, which were not differentially expressed at the mRNA level, were differentially expressed, suggesting that the expression of the two may be regulated by translation or post-translation level in BC. In conclusion, ORC1L, ORC5L and ORC6L were differentially expressed in BC tissues and may be involved in the occurrence and development of BC.

\section{Relationship between ORCs and clinicopathological characteristics in BC}

We further excavated the relationship between the expression of ORC1L, ORC5L, and ORC6L and the clinicopathological characteristics of BC patients using TCGA-BRCA data, including tumor patient age, molecular subtype, tumor volume, lymph node metastasis, distant metastasis, and pathological stage. The results suggested that the high expression of ORC1L and ORC6L were mainly Her2 overexpressed subtype, and their expression were negatively correlated with patient age and positively correlated with tumor size, but not with lymph node metastasis, distant metastasis and tumor stage (Fig.3A, C). The expression of ORC5L was also negatively correlated with age and positively correlated with lymph node metastasis, but not with BC molecular subtype, tumor size and other characteristics (Fig.3B).

\section{Diagnostic and prognostic values of ORC1L, ORC5L and ORC6L in BC}

ROC curves were used to evaluate the diagnostic value of ORC1L, ORC5L, and ORC6L in BC. The results showed that the area under the curve (AUC) of ORC1L was $0.895,95(\mathrm{Cl})=0.869-0.921$ and that of ORC5L was $0.763,95(\mathrm{Cl})=0.722-0.805$. Of concern is that ORC6L had the highest diagnostic value and the AUC was $0.910,95(\mathrm{Cl})=0.887$ to 0.934 (Fig. $4 \mathrm{~A})$.

Then, Kaplan-Meier method was used to illustrate the impacts of ORC1L, ORC5L, and ORC6L on patient survival. Interestingly, the expression of ORC1L, ORC5L, and ORC6L were not significantly correlated with OS in BC patients (Fig.4B). It was inconsistent with previous results that ORC5L and ORC6L have prognostic value based on KM-plot online analysis. Since the expression of ORC1L, ORC5L, and ORC6L were related to clinicopathological factors such as age and molecular subtype, a univariate and multivariate Cox proportional risk regression model was performed on OS to eliminate the interference of the above factors. Univariate analysis showed that T stage, $\mathrm{N}$ stage, M stage, pathological stage, molecular subtype, and age were significantly associated with OS. Further multivariate regression analysis was performed. We found that ORC6L, as well as $\mathrm{N}$ stage, $\mathrm{M}$ stage, pathological stage, molecular subtype and age, were determined to be independent risk factors affecting OS (Table 2, Fig.5A). The risk of death of ORC6L high-expression patients was 1.538-fold of those patients with low ORC6L expression.

We included all statistically significant factors in the multivariate Cox proportional risk regression model, including $\mathrm{N}$ stage, $\mathrm{M}$ stage, pathological stage, molecular subtype, age, and ORC6L expression, to construct a nomogram for predicting the 1-year, 3-year, and 5-year survival probability of $\mathrm{BC}$ patients (Fig. 5B). The C-index calculated by internal re-sampling verification is $0.716,95 \% \mathrm{Cl}(0.692-0.741)$, indicating that the predicted results were in good agreement with the real results. The calibration plot also indicates that the nomogram was well calibrated, with mean predicted probabilities close to observed probabilities (Fig. 5C). In conclusion, ORC6L had good clinical application value and prospect.

\section{Enrichment analysis of ORC6L related pathways in BC}

Based on the striking expression patterns observed, a GSEA was performed to further illustrate the molecular role of ORC6L in BC. According to the median value of ORC6L mRNA expression in BC, patients from TCGA were divided into high and low ORC6L expression groups. GSEA revealed that 14 gene sets were strongly enriched in ORC6L high BC specimens compared with ORC6L low specimens (Fig.6A, Table 3). Some of these gene sets, such as cell cycle checkpoints and cellular senescence, were markedly activated in the ORC6L high BC specimens in this study, implying potential cell cycle regulatory mechanism of ORC6L in BC. Pathways that modulate cancer-related functions, such as transcriptional regulation by TP53, fceri mediated NF-KB activation, and TCF dependent signaling in response to WNT, were positively correlated with ORC6L expression. In addition, GSEA revealed a relationship between the ORC6L expression and activation of RHO GTPase effectors, signaling by the B cell receptor BCR, and epigentic regulation of gene expression. Together, these results suggested that ORC6L may play an oncogenic role in $\mathrm{BC}$ by regulating the cell cycle and activating tumor-related signaling pathways. 
Tumor growth and development is determined by both cancer cell-autonomous and microenvironmental mechanisms, including tumor-infiltrating immune cells. Accumulated evidence has shown that tumor infiltrating lymphocytes play an important role in the development and progression of $\mathrm{BC}^{23,24}$. Therefore, the ssGSEA of the GSVA package ${ }^{25}$ was used to determine whether ORC6L modulates the tumor microenvironment. We found that ORC6L was significantly related to immune infiltrations in BC. In particular, there was a strong positive correlation of Th2 cell, Treg cell『as well as a DC cell and Th1 cell with ORC6L expression level in BC. It is worth noting that strong ORC6L expression was closely negative correlated with mast cell, NK cell, eosinophils, and Th17 cell levels in BC. These results suggested that ORC6L may be involved in the occurrence and progression of $\mathrm{BC}$ by regulating immune cells infiltration.

\section{Discussion}

ORC is known for its role in regulating the initiation of DNA replication. Its role in tumors has attracted increasing attention in recent years. Previous study showned that ORC1L was up-regulated in gliomas and promoted proliferation, migration and invasion of glioma cells through activation of ERK/JNK signaling pathway ${ }^{10}$. In lung cancer and triple-negative BC, ORC1L was considered as a pivotal gene for the maintenance of tumor stem cell, and its expression in lung cancer was positively correlated with tumor stage ${ }^{19,20}$. ORC5L and ORC6L had prognostic value in hepatocellular carcinoma ${ }^{17}$. It was reported that ORCs were also associated with chemotherapy resistance of various tumors. Upregulation of ORC2L and ORC5L had been detected in cisplatin-resistant bladder cancer cell lines ${ }^{22}$. In pancreatic cancer, ORC4L was involved in PIK1-mediated gemcitabine resistance ${ }^{21}$. In colorectal cancer cells, high expressed ORC6L promoted cell proliferation and induced 5-FU and cisplatin resistance ${ }^{13}$. Therefore, the ORCs had potential clinical application value in a variety of tumors.

In this study, we systematically analyzed the expression, diagnostic and prognostic value of the ORCs in BC for the first time. We demonstrated that mRNA and protein expression of ORC1L and ORC6L were significantly upregulated in BC tissues compared with normal tissues by analyzing Oncomine, TCGA, GEO and ULCAN databases. In addition, both ORC1L and ORC6L had high diagnostic value in BC. Kaplan-Meier method found that ORCs were not associated with OS in BC patients, which was inconsistent with Kaplan-Meier Plotter's prediction. Age and molecular subtype might affect the effect of ORCs on OS due to clinical correlation analysis found the ORCs expression were correlated with above factors. Therefore, we included clinicopathological factors in the multivariate COX regression analysis and finally confirmed that ORC6L is an independent prognostic factor for BC. ORC1L and ORC5L had no significance for OS although they were expressed differently in BC.

Accumulated evidence suggest that ORC6L is a potential oncogenic gene in BC and can be used as a potential prognostic molecule and novel therapeutic target to improve the survival of $B C$ patients. However, the expression of ORC5L in $B C$ is controversial and its diagnostic value is relatively low. More evidence is needed to confirm its expression in BC. The ORC2L and ORC3L proteins were abnormally expressed in BC but had no significant differences in mRNA levels and had no effect on OS. ORC4L was not differentially expressed at either the protein or mRNA level. In view of the significant expression and clinical significance of ORC6L, we incorporated ORC6L and OS-related clinicopathological indicators to construct a nomogram for predicting the probability of survival of BC patients at 1, 3, and 5 years, which was found to be highly accurate and of high clinical application value.

Comparison of the transcriptional profile with GSEA led us to identify a cohort of pathways (such as cycle checkpoints and cellular senescence) that were activated in the ORC6L higher expression BC tissues. Besides, pathways such as TP53, NF-KB and WNT were also activated. The products of these pathways promote proliferation, angiogenesis, stem cell characteristics and tumor migration. However, the exact molecular mechanism of ORC6L needs to be validated in clinical BC specimens and animal models in the future.

Cumulative evidence advocates that tumor-infiltrating immune cells play essential roles in BC development and progression ${ }^{26}$. Th1 and Th2 cytokines were shown to contribute to tumorigenesis in several models. Functional Th1-oriented T follicular helper tumor infiltrating lymphocytes that infiltrate human $\mathrm{BC}$ promoted effective adaptive immunity ${ }^{27}$.Th2 activation was associated with poor prognosis in human $\mathrm{BC}^{28}$. Recently, Ghirelli et al. described a novel GM-CSF/pDC/Th2 axis with aggressive disease subtypes in $\mathrm{BC}^{29}$. Treg cells, a cell type critical for anti-tumor immunity, play a major role in the development of an immunosuppressive tumor microenvironment. Studies have shown that increased numbers of tumor-infiltrating Tregs correlate with reduced survival, and quantification of Tregs enables the identification of high-risk BC patients and those at risk of late relapse ${ }^{30}$.We found that the high expression of ORC6L was associated with the increased infiltration of Th1, Th2 and Treg cells, and speculated that ORC6L might improve the composition ratio of the above immune cells infiltration in BC through some mechanism, thus affecting tumor progression and clinical prognosis.

The protumoral and antitumoral role of mast cells in BC progression is still controversial ${ }^{31}$. Studies showed that mast cells increased BC growth and metastasis ${ }^{32}$, but the study by della Rovere et al. revealed that peritumoral mast cells seemed to have a cytolytic activity against tumor cells ${ }^{33}$. Moreover, decreasing NK-cell tumour immunosurveillance was found to enhance metastasis in BC models ${ }^{34}$. We found that infiltration of mast cells and NK cells was reduced in BC tissues with high ORC6L expression, suggesting that inhibition of tumor infiltration by ORC6L is another factor that promotes BC progression. Further studies will be needed to evaluate the relative role of these immune cells within the BC microenvironment.

\section{Conclusions}


We demonstrated for the first time that ORC1L, ORC5L and ORC6L are differentially expressed in BC and have good diagnostic value. Among them, ORC6L has the highest diagnostic value and is an independent risk factor that affects the OS of BC patients and can be used as a potential prognostic biomarker. Since the expression, diagnosis and prognostic value of ORCs in BC were based on public databases, more experiments and clinical studies are needed.

\section{Methods}

\section{Kaplan-Meier Plotter (KM-Plotter)}

The Kaplan Meier plotter (http://kmplot.com/analysis/) is capable of assessing the effects of more than 54000 genes (mRNA, miRNA, protein) on survival in 21 cancer types including breast $(n=7,830)$, ovarian $(n=2,190)$, lung $(n=3,452)$, and gastric $(n=1,440)$ cancer. The sources for the databases were GEO, EGA, and TCGA. It is a meta-analysis based discovery and validation of survival biomarkers ${ }^{35}$. We assessed the association between ORCs and overall survival(OS) in BC patients via Kaplan-Meier Plotter. P-value $<0.05$ was considered statistically significant.

\section{Gene expression and clinical information in BC}

The ORCs mRNA expression levels were analyzed in BC and normal tissues based on the TCGA database (https://portal.gdc.cancer.gov/), GEO database (accession numbers GSE54002 and GSE37751) (https://www.ncbi.nlm.nih.gov/geo/), and Oncomine platform (www.oncomine.org). We obtained gene expression data and clinical information of 1109 female BC cases and 113 normal breast tissues from TCGA. The RNAseq data in FPKM (Fregments Per Kilobase Per Million) format were converted to TPM (Transcripts Per Million reads) format and log2 converted for subsequent analysis. The clinical characteristics of $\mathrm{BC}$ are present in Table1.

\section{Protein Expression Analysis of ORCs in BC}

UALCAN is a comprehensive and interactive web resource for cancer OMICS data analysis (http://ualcan.path.uab.edu/). It is built on PERL-CGI with high quality graphics using javascript and CSS. One of the user-friendly features of portal is that it allows analysis of protein expression analysis option using data from the Clinical Proteomic Tumor Analysis Consortium (CPTAC) Confirmatory/Discovery dataset ${ }^{36}$. We explored the protein expression of the ORCs in BC via UALCAN by CPTAC analysis.

\section{Construction and verification of nomogram}

The identified independent factors associated with BC prognosis were used to construct a nomogram that predicted the probability of 1-, 3-, and 5year OS. Nomogram was formulated by $\mathrm{R}$ with the rms and survival package ${ }^{37}$. The Harrell's Concordance index (C-index) was used to quantify the predictive accuracy, which ranges from 0.5 (no predictive power) to 1 (perfect prediction). In addition, calibration plots were generated to examine the performance characteristics of the predictive nomogram.

\section{Gene set enrichment analysis (GSEA)}

GSEA (http://www.broadinstitute.org/gsea/index.jsp) is a tool that determines whether a set of genes show statistically significant, concordant differences between two biological states ${ }^{38}$. Publicly available microarray expression data of 1109 BC samples were downloaded from TCGA. GSEA was used to associate gene signatures with ORC6L expression status (ORC6L low vs. ORC6L high). The significance threshold was set at a nominal $P$-value $<0.05$ and false discovery rate $(F D R)<0.25$.

\section{Correlation analysis of immune cell infiltration}

The relative tumor infiltration levels of 24 kinds of immune cell type were quantified by ssGSEA of GSVA package $\mathrm{e}^{25}$. The correlation between risk scores and immune infiltration was calculated using Spearman Correlation Test. Coefficient of correlation |r|ख0.3凶P-value $<0.05$ was considered statistically significant.

\section{Statistical analysis}

Statistical analysis and mapping were performed using R (V.3.6.3). Wilcoxon rank sum test was used to compare the difference of gene expression between $\mathrm{BC}$ and normal breast tissues and the correlation between gene expression and clinicopathological features. Receiver operating characteristic (ROC) curves were plotted using pROC package. Kaplan-meier and Cox proportional risk regression model were used to evaluate the prognostic value of ORCs. P-value $<0.05$ was considered statistically significant.

\section{Abbreviations}

AUC: area under the curve

BC: breast cancer 
Cl: confidence interval

HR: hazard ratio

ORC: origin recognition complex

OS: overall survival

ROC: receiver operating characteristic

\section{Declarations}

\section{Ethics approval and consent to participate}

Not applicable.

\section{Consent for publication}

Not applicable.

\section{Availability of data and materials}

The raw data of this study are derived from TCGA database (https://portal.gdc.cancer.gov/), GEO database (https://www.ncbi.nlm.nih.gov/geo/) and Oncomine database (www.oncomine.org), which are publicly available databases.

\section{Competing interests}

The authors declare that they have no competing interests.

\section{Funding}

This research was supported by National Natural Science Foundation of China (No.81802478), Guangxi Natural Science Foundation (No.2018GXNSFBA050042) and Guangxi Science and technology base and talents special project (No.AD20238005).

\section{Authors' contributions}

SC wrote this manuscript. SC, ZJ and LX analyzed and interpreted the data. LL, XZ and YG participated in this study. JL designed and supervised this study. All authors read and approved the final manuscript.

\section{Acknowledgements}

Not applicable.

\section{References}

1. Sung H, Ferlay J, Siegel RL, Laversanne M, Soerjomataram I, Jemal A, et al. Global Cancer Statistics 2020: GLOBOCAN Estimates of Incidence and Mortality Worldwide for 36 Cancers in 185 Countries. CA Cancer J Clin. 2021; 71(3):209-249.

2. Cardoso F, Fallowfield L, Costa A, Castiglione M, Senkus E. Locally recurrent or metastatic breast cancer: ESMO Clinical Practice Guidelines for diagnosis, treatment and follow-up. Annals of oncology : official journal of the European Society for Medical Oncology. 2011; 22 Suppl 6:vi25-30.

3. Bell SP, Dutta A. DNA replication in eukaryotic cells. Annual review of biochemistry. 2002; 71:333-374.

4. Prasanth SG, Shen Z, Prasanth KV, Stillman B. Human origin recognition complex is essential for HP1 binding to chromatin and heterochromatin organization. Proceedings of the National Academy of Sciences of the United States of America. 2010; 107(34):15093-15098.

5. Prasanth SG, Prasanth KV, Stillman B. Orc6 involved in DNA replication, chromosome segregation, and cytokinesis. Science (New York, NY). 2002; 297(5583):1026-1031.

6. Dhar SK, Yoshida K, Machida Y, Khaira P, Chaudhuri B, Wohlschlegel JA, et al. Replication from oriP of Epstein-Barr virus requires human ORC and is inhibited by geminin. Cell. 2001; 106(3):287-296.

7. Kordon MM, Szczurek A, Berniak K, Szelest O, Solarczyk K, Tworzydło M, et al. PML-like subnuclear bodies, containing XRCC1, juxtaposed to DNA replication-based single-strand breaks. FASEB journal : official publication of the Federation of American Societies for Experimental Biology. 2019; 
8. Koleck TA, Bender CM, Clark BZ, Ryan CM, Ghotkar P, Brufsky A, et al. An exploratory study of host polymorphisms in genes that clinically characterize breast cancer tumors and pretreatment cognitive performance in breast cancer survivors. Breast cancer (Dove Medical Press). 2017; 9:95-110.

9. El-Saghire H, Vandevoorde C, Ost P, Monsieurs P, Michaux A, De Meerleer G, et al. Intensity modulated radiotherapy induces pro-inflammatory and pro-survival responses in prostate cancer patients. International journal of oncology. 2014; 44(4):1073-1083.

10. Xiong W, Xie C, Qiu Y, Tu Z, Gong Q. Origin recognition complex subunit 1 regulates cell growth and metastasis in glioma by altering activation of ERK and JNK signaling pathway. Molecular and cellular probes. 2020; 49:101496.

11. Liu Y, Chen P, Li M, Fei H, Huang J, Zhao T, et al. Comprehensive Analysis of the Control of Cancer Stem Cell Characteristics in Endometrial Cancer by Network Analysis. Computational and mathematical methods in medicine. 2021; 2021:6653295.

12. Gavin EJ, Song B, Wang Y, Xi Y, Ju J. Reduction of Orc6 expression sensitizes human colon cancer cells to 5-fluorouracil and cisplatin. PloS one. 2008; 3(12):e4054.

13. Xi Y, Formentini A, Nakajima G, Kornmann M, Ju J. Validation of biomarkers associated with 5-fluorouracil and thymidylate synthase in colorectal cancer. Oncology reports. 2008; 19(1):257-262.

14. Hu Y, Wang L, Li Z, Wan Z, Shao M, Wu S, et al. Potential Prognostic and Diagnostic Values of CDC6, CDC45, ORC6 and SNHG7 in Colorectal Cancer. OncoTargets and therapy. 2019; 12:11609-11621.

15. Mao R, Wang Z, Zhang Y, Chen Y, Liu Q, Zhang T, et al. Development and validation of a novel prognostic signature in gastric adenocarcinoma. Aging. 2020; 12(21):22233-22252.

16. Zhou W, Li J, Lu X, Liu F, An T, Xiao X, et al. Derivation and Validation of a Prognostic Model for Cancer Dependency Genes Based on CRISPRCas9 in Gastric Adenocarcinoma. Frontiers in oncology. 2021; 11:617289.

17. Wang XK, Wang QQ, Huang JL, Zhang LB, Zhou X, Liu JQ, et al. Novel candidate biomarkers of origin recognition complex 1,5 and 6 for survival surveillance in patients with hepatocellular carcinoma. Journal of Cancer. 2020; 11(7):1869-1882.

18. Nangraj AS, Selvaraj G, Kaliamurthi S, Kaushik AC, Cho WC, Wei DQ. Integrated PPI- and WGCNA-Retrieval of Hub Gene Signatures Shared Between Barrett's Esophagus and Esophageal Adenocarcinoma. Frontiers in pharmacology. 2020; 11:881.

19. Zhang Y, Tseng JT, Lien IC, Li F, Wu W, Li H. mRNAsi Index: Machine Learning in Mining Lung Adenocarcinoma Stem Cell Biomarkers. Genes. 2020; 11(3).

20. Suo HD, Tao Z, Zhang L, Jin ZN, Li XY, Ma W, et al. Coexpression Network Analysis of Genes Related to the Characteristics of Tumor Stemness in Triple-Negative Breast Cancer. BioMed research international. 2020; 2020:7575862.

21. Song B, Liu XS, Rice SJ, Kuang S, Elzey BD, Konieczny SF, et al. PIk1 phosphorylation of orc2 and hbo1 contributes to gemcitabine resistance in pancreatic cancer. Molecular cancer therapeutics. 2013; 12(1):58-68.

22. Kim SH, Ho JN, Jin H, Lee SC, Lee SE, Hong SK, et al. Upregulated expression of BCL2, MCM7, and CCNE1 indicate cisplatin-resistance in the set of two human bladder cancer cell lines: T24 cisplatin sensitive and T24R2 cisplatin resistant bladder cancer cell lines. Investigative and clinical urology. 2016; 57(1):63-72.

23. Deepak KGK, Vempati R, Nagaraju GP, Dasari VR, S N, Rao DN, et al. Tumor microenvironment: Challenges and opportunities in targeting metastasis of triple negative breast cancer. Pharmacological research. 2020; 153:104683.

24. Mittal S, Brown NJ, Holen I. The breast tumor microenvironment: role in cancer development, progression and response to therapy. Expert review of molecular diagnostics. 2018; 18(3):227-243.

25. Hänzelmann S, Castelo R, Guinney J. GSVA: gene set variation analysis for microarray and RNA-seq data. BMC bioinformatics. 2013 ; $14: 7$.

26. Stanton SE, Disis ML. Clinical significance of tumor-infiltrating lymphocytes in breast cancer. Journal for immunotherapy of cancer. 2016 ; $4: 59$.

27. Noël G, Langouo Fontsa M, Garaud S, De Silva P, de Wind A, Van den Eynden GG, et al. Functional Th1-oriented T follicular helper cells that infiltrate human breast cancer promote effective adaptive immunity. The Journal of clinical investigation. 2021. 
28. Teschendorff AE, Gomez S, Arenas A, El-Ashry D, Schmidt M, Gehrmann M, et al. Improved prognostic classification of breast cancer defined by antagonistic activation patterns of immune response pathway modules. BMC cancer. 2010; 10:604.

29. Ghirelli C, Reyal F, Jeanmougin M, Zollinger R, Sirven P, Michea P, et al. Breast Cancer Cell-Derived GM-CSF Licenses Regulatory Th2 Induction by Plasmacytoid Predendritic Cells in Aggressive Disease Subtypes. Cancer research. 2015; 75(14):2775-2787.

30. Bates GJ, Fox SB, Han C, Leek RD, Garcia JF, Harris AL, et al. Quantification of regulatory T cells enables the identification of high-risk breast cancer patients and those at risk of late relapse. Journal of clinical oncology : official journal of the American Society of Clinical Oncology. 2006; 24(34):5373-5380.

31. Aponte-López A, Fuentes-Pananá EM, Cortes-Muñoz D, Muñoz-Cruz S. Mast Cell, the Neglected Member of the Tumor Microenvironment: Role in Breast Cancer. Journal of immunology research. 2018; 2018:2584243.

32. Majorini MT, Cancila V, Rigoni A, Botti L, Dugo M, Triulzi T, et al. Infiltrating Mast Cell-Mediated Stimulation of Estrogen Receptor Activity in Breast Cancer Cells Promotes the Luminal Phenotype. Cancer research. 2020; 80(11):2311-2324.

33. della Rovere F, Granata A, Familiari D, D'Arrigo G, Mondello B, Basile G. Mast cells in invasive ductal breast cancer: different behavior in high and minimum hormone-receptive cancers. Anticancer research. 2007; 27(4b):2465-2471.

34. Bottos A, Gotthardt D, Gill JW, Gattelli A, Frei A, Tzankov A, et al. Decreased NK-cell tumour immunosurveillance consequent to JAK inhibition enhances metastasis in breast cancer models. Nature communications. 2016; 7:12258.

35. Ősz Á, Lánczky A, Győrffy B. Survival analysis in breast cancer using proteomic data from four independent datasets. Scientific reports. 2021; 11(1):16787.

36. Chandrashekar DS, Bashel B, Balasubramanya SAH, Creighton CJ, Ponce-Rodriguez I, Chakravarthi B, et al. UALCAN: A Portal for Facilitating Tumor Subgroup Gene Expression and Survival Analyses. Neoplasia (New York, NY). 2017; 19(8):649-658.

37. Liu J, Lichtenberg T, Hoadley KA, Poisson LM, Lazar AJ, Cherniack AD, et al. An Integrated TCGA Pan-Cancer Clinical Data Resource to Drive High-Quality Survival Outcome Analytics. Cell. 2018; 173(2):400-416.e411.

38. Subramanian A, Tamayo P, Mootha VK, Mukherjee S, Ebert BL, Gillette MA, et al. Gene set enrichment analysis: a knowledge-based approach for interpreting genome-wide expression profiles. Proceedings of the National Academy of Sciences of the United States of America. 2005; 102(43):15545-15550.

\section{Tables}

Table 1 The clinical characteristics of BC patients (TCGA). 


\begin{tabular}{|c|c|c|c|}
\hline Characteristic & Low expression of ORC6L & High expression of ORC6L & $P$ value \\
\hline$n$ & 541 & 542 & \\
\hline T stage, $\mathrm{n}(\%)$ & & & $<0.001$ \\
\hline T1 & $162(15 \%)$ & $115(10.6 \%)$ & \\
\hline T2 & $281(26 \%)$ & $348(32.2 \%)$ & \\
\hline T3 & $78(7.2 \%)$ & $61(5.6 \%)$ & \\
\hline $\mathrm{T} 4$ & $20(1.9 \%)$ & $15(1.4 \%)$ & \\
\hline N stage, $\mathrm{n}(\%)$ & & & 0.016 \\
\hline NO & $261(24.5 \%)$ & $253(23.8 \%)$ & \\
\hline N1 & $173(16.3 \%)$ & $185(17.4 \%)$ & \\
\hline N2 & $50(4.7 \%)$ & $66(6.2 \%)$ & \\
\hline N3 & $50(4.7 \%)$ & $26(2.4 \%)$ & \\
\hline M stage, n (\%) & & & 1 \\
\hline MO & $434(47.1 \%)$ & $468(50.8 \%)$ & \\
\hline M1 & $10(1.1 \%)$ & $10(1.1 \%)$ & \\
\hline Pathologic stage, n (\%) & & & 0.036 \\
\hline Stage I & $105(9.9 \%)$ & $76(7.2 \%)$ & \\
\hline Stage II & $287(27.1 \%)$ & $332(31.3 \%)$ & \\
\hline Stage III & $126(11.9 \%)$ & $116(10.9 \%)$ & \\
\hline Stage IV & $10(0.9 \%)$ & $8(0.8 \%)$ & \\
\hline Age, n (\%) & & & $<0.001$ \\
\hline$<=60$ & $272(25.1 \%)$ & $329(30.4 \%)$ & \\
\hline$>60$ & $269(24.8 \%)$ & $213(19.7 \%)$ & \\
\hline Histological type, n (\%) & & & $<0.001$ \\
\hline Infiltrating Ductal Carcinoma & $315(32.2 \%)$ & $457(46.8 \%)$ & \\
\hline Infiltrating Lobular Carcinoma & $172(17.6 \%)$ & $33(3.4 \%)$ & \\
\hline PAM50, n (\%) & & & $<0.001$ \\
\hline Normal & $28(2.6 \%)$ & $12(1.1 \%)$ & \\
\hline LumA & $437(40.4 \%)$ & $125(11.5 \%)$ & \\
\hline LumB & $52(4.8 \%)$ & $152(14 \%)$ & \\
\hline Her2 & $16(1.5 \%)$ & $66(6.1 \%)$ & \\
\hline Basal & $8(0.7 \%)$ & $187(17.3 \%)$ & \\
\hline OS event, n (\%) & & & 0.194 \\
\hline Alive & $473(43.7 \%)$ & $458(42.3 \%)$ & \\
\hline Dead & $68(6.3 \%)$ & $84(7.8 \%)$ & \\
\hline Age, meidan (IQR) & $60(49,69)$ & $56(48,65.75)$ & $<0.001$ \\
\hline
\end{tabular}

Table2 Univariate and multivariate Cox regression for OS in BC patients (TCGA). 


\begin{tabular}{|c|c|c|c|c|c|}
\hline \multirow[t]{2}{*}{ Characteristics } & \multirow{2}{*}{$\begin{array}{l}\text { Total } \\
(\mathrm{N})\end{array}$} & \multicolumn{2}{|l|}{ Univariate analysis } & \multicolumn{2}{|l|}{ Multivariate analysis } \\
\hline & & $\mathrm{HR}(95 \% \mathrm{Cl})$ & P val & $\mathrm{HR}(95 \% \mathrm{Cl})$ & P val \\
\hline T stage & 1079 & & & & \\
\hline T1 & 277 & Reference & & & \\
\hline T2\&T3\&T4 & 803 & $1.482(1.007-2.182)$ & 0.046 & $1.097(0.695-1.730)$ & 0.691 \\
\hline N stage & 1063 & & & & \\
\hline NO & 514 & Reference & & & \\
\hline N1\&N2\&N3 & 550 & 2.239 (1.567-3.199) & $<0.001$ & $1.688(1.080-2.638)$ & 0.022 \\
\hline M stage & 922 & & & & \\
\hline M0 & 902 & Reference & & & \\
\hline M1 & 20 & $4.254(2.468-7.334)$ & $<0.001$ & $2.469(1.213-5.028)$ & 0.013 \\
\hline Pathologic stage & 1059 & & & & \\
\hline Stage I\&Stage II & 800 & Reference & & & \\
\hline Stage III\&Stage IV & 260 & 2.391 (1.703-3.355) & $<0.001$ & $1.785(1.127-2.828)$ & 0.013 \\
\hline Age & 1082 & & & & \\
\hline$<=60$ & 601 & Reference & & & \\
\hline$>60$ & 482 & $2.020(1.465-2.784)$ & $<0.001$ & 2.303 (1.591-3.335) & $<0.001$ \\
\hline Histological type & 977 & & & & \\
\hline Infiltrating Ductal Carcinoma & 772 & Reference & & & \\
\hline Infiltrating Lobular Carcinoma & 205 & $0.827(0.526-1.299)$ & 0.41 & & \\
\hline PAM50 & 1082 & & & & \\
\hline Her2 & 82 & Reference & & & \\
\hline LumA\&LumB\&Basal\&Normal & 1001 & $0.528(0.318-0.875)$ & 0.013 & $0.555(0.314-0.981)$ & 0.043 \\
\hline ORC1 & 1082 & & & & \\
\hline Low & 542 & Reference & & & \\
\hline High & 541 & $1.206(0.877-1.659)$ & 0.249 & & \\
\hline ORC5 & 1082 & & & & \\
\hline Low & 542 & Reference & & & \\
\hline High & 541 & $0.921(0.670-1.268)$ & 0.615 & & \\
\hline ORC6 & 1082 & & & & \\
\hline Low & 542 & Reference & & & \\
\hline High & 541 & $1.270(0.922-1.749)$ & 0.143 & $1.538(1.060-2.231)$ & 0.023 \\
\hline
\end{tabular}

Note: $\mathrm{HR}(95 \% \mathrm{Cl})$ :Hazard ratio $(95 \% \mathrm{Cl})$

Table 3 Signaling pathways enriched in the ORC6L high-expression group in BC patients (TCGA). 


\begin{tabular}{|c|c|c|c|c|c|}
\hline Signaling pathway & $\begin{array}{l}\text { Set } \\
\text { Size }\end{array}$ & ES & NES & $\begin{array}{l}\mathrm{p} \\
\text { value }\end{array}$ & $\begin{array}{l}q \\
\text { value }\end{array}$ \\
\hline FCERI_MEDIATED_NF_KB_ACTIVATION & 136 & 0.654 & 1.888 & 0.005 & 0.004 \\
\hline CELL_CYCLE_CHECKPOINTS & 291 & 0.623 & 1.858 & 0.005 & 0.004 \\
\hline SIGNALING_BY_THE_B_CELL_RECEPTOR_BCR_ & 166 & 0.630 & 1.840 & 0.005 & 0.004 \\
\hline S_PHASE & 161 & 0.618 & 1.802 & 0.005 & 0.004 \\
\hline IMMUNOREGULATORY_INTERACTIONS_BETWEEN_A_LYMPHOID_AND_A_NON_LYMPHOID_CELL & 186 & 0.604 & 1.773 & 0.005 & 0.004 \\
\hline M_PHASE & 414 & 0.562 & 1.687 & 0.005 & 0.004 \\
\hline CELL_SURFACE_INTERACTIONS_AT_THE_VASCULAR_WALL & 194 & 0.555 & 1.633 & 0.005 & 0.004 \\
\hline EPIGENETIC_REGULATION_OF_GENE_EXPRESSION & 146 & 0.543 & 1.574 & 0.013 & 0.011 \\
\hline RHO_GTPASE_EFFECTORS & 322 & 0.514 & 1.536 & 0.005 & 0.004 \\
\hline DNA_REPAIR & 330 & 0.513 & 1.533 & 0.005 & 0.004 \\
\hline NATURAL_KILLER_CELL_MEDIATED_CYTOTOXICITY & 132 & 0.520 & 1.496 & 0.039 & 0.033 \\
\hline CELLULAR_SENESCENCE & 194 & 0.492 & 1.448 & 0.030 & 0.026 \\
\hline TCF_DEPENDENT_SIGNALING_IN_RESPONSE_TO_WNT & 231 & 0.477 & 1.412 & 0.040 & 0.034 \\
\hline TRANSCRIPTIONAL_REGULATION_BY_TP53 & 358 & 0.452 & 1.354 & 0.023 & 0.020 \\
\hline
\end{tabular}

Note: P value < 0.05 and FDR q value< 0.25 were considered significantly enriched; p value was adjusted. ES: Enrichment Score; NES: normalized enrichment score.

\section{Figures}



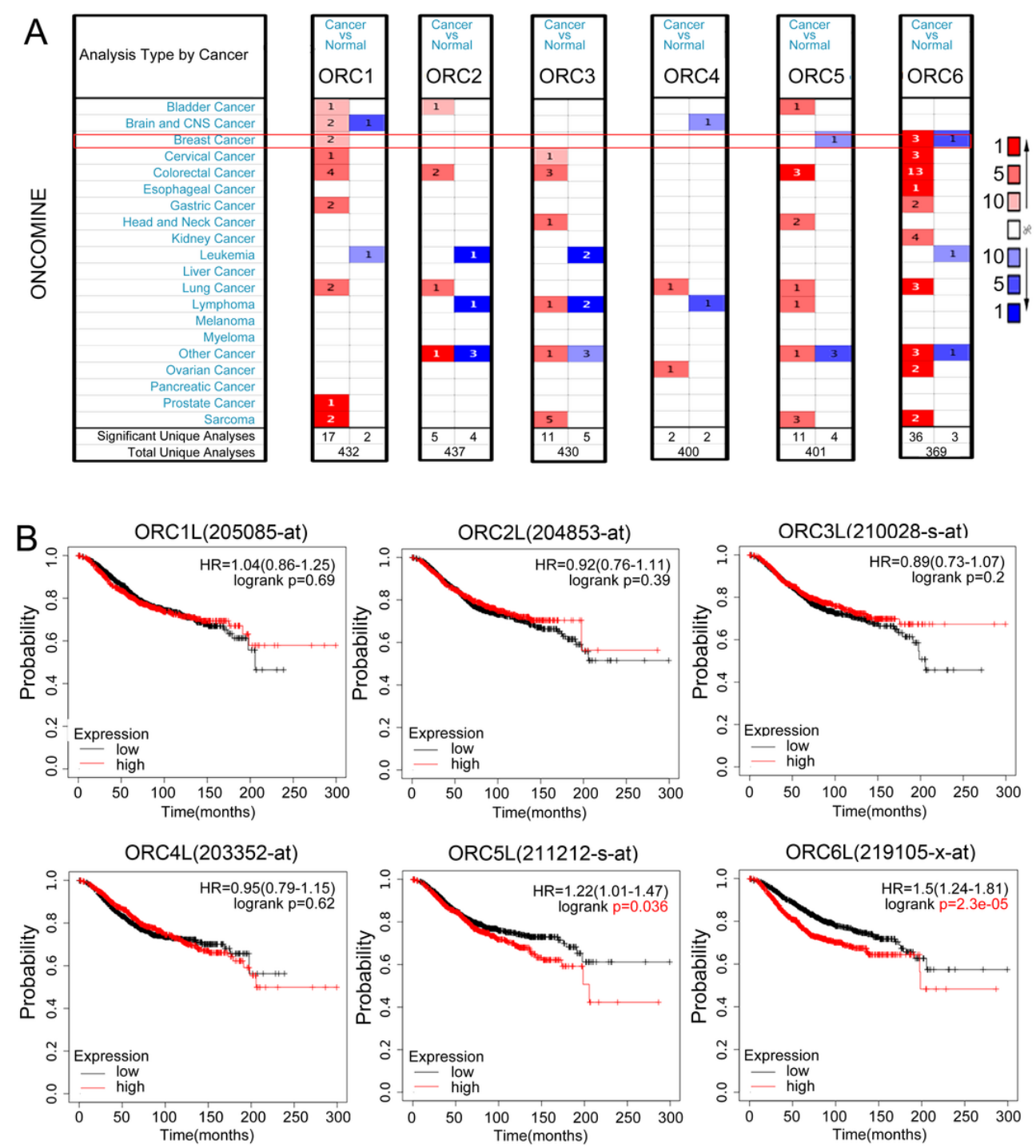

Figure 1

Expression of ORCs in various tumors and their prognostic value in BC (A)Comprehensive analysis of ORCs mRNA expression in various tumor tissues compared with normal tissues using Oncomine database. The left box in red indicated the number of datasets with high expression and the right box in blue indicated the number of datasets with low expression after comparing cancerous and normal tissues. (B)The Kaplan-Meier curve for OS of BC patients with high and low ORCs mRNA expression from Kaplan-Meier Plotter. 

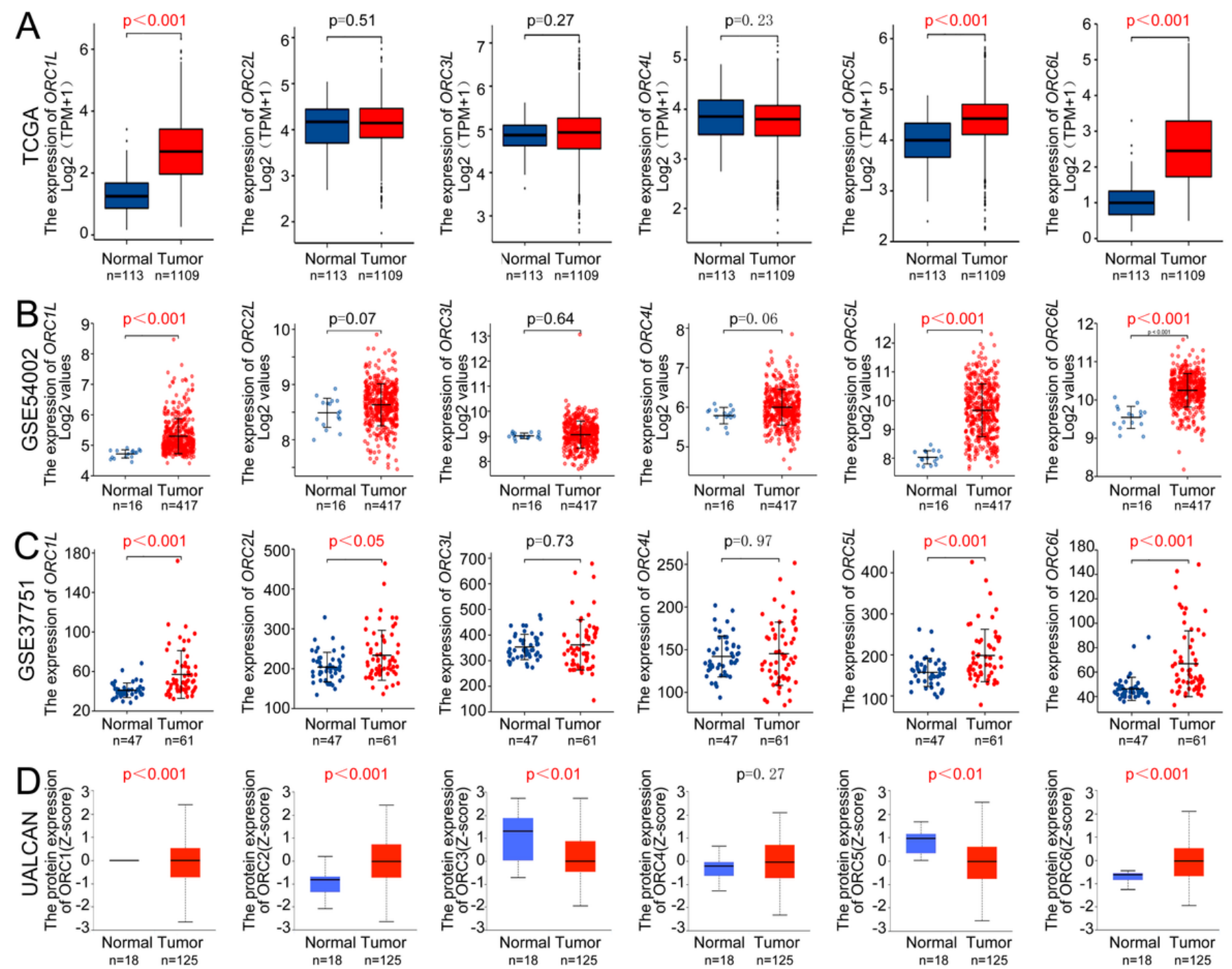

Figure 2

mRNA and protein expression of ORCs in BC (A) Relative mRNA expression of ORCs in human BC and normal tissues obtained from TCGA. (B-C) Relative mRNA expression of ORCs in human BC and normal tissues obtained from GEO (GSE54002, GSE37751). (D) ORCs protein expression in $\mathrm{BC}$ and normal tissues from UALCAN cancer OMICS data. $\mathrm{n}=$ number of cases. P-value $<0.05$ was considered statistically significant. 

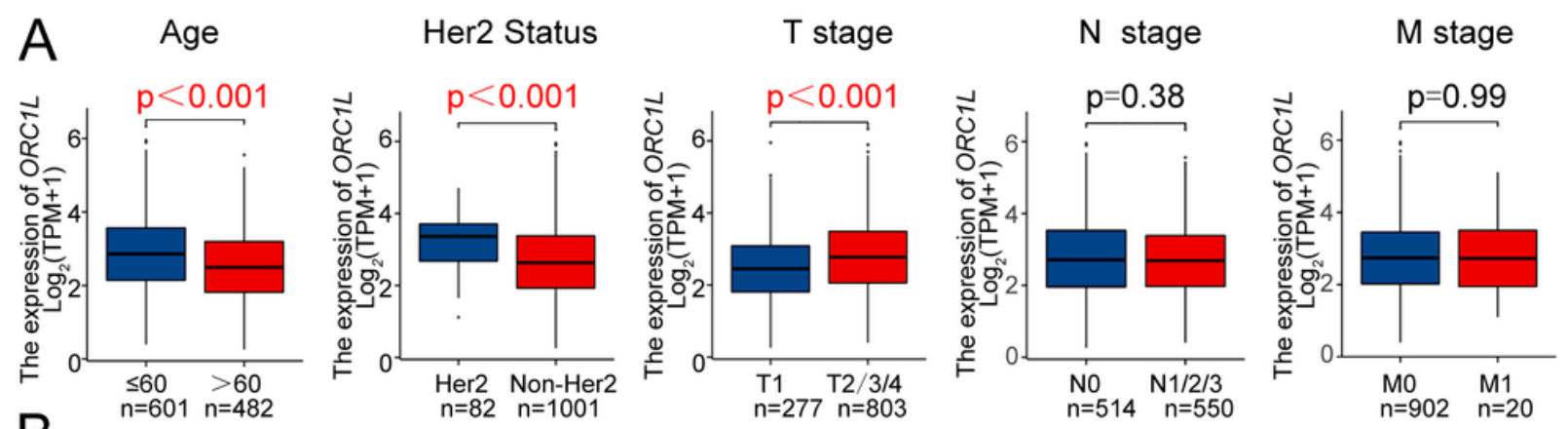

pathologic stage
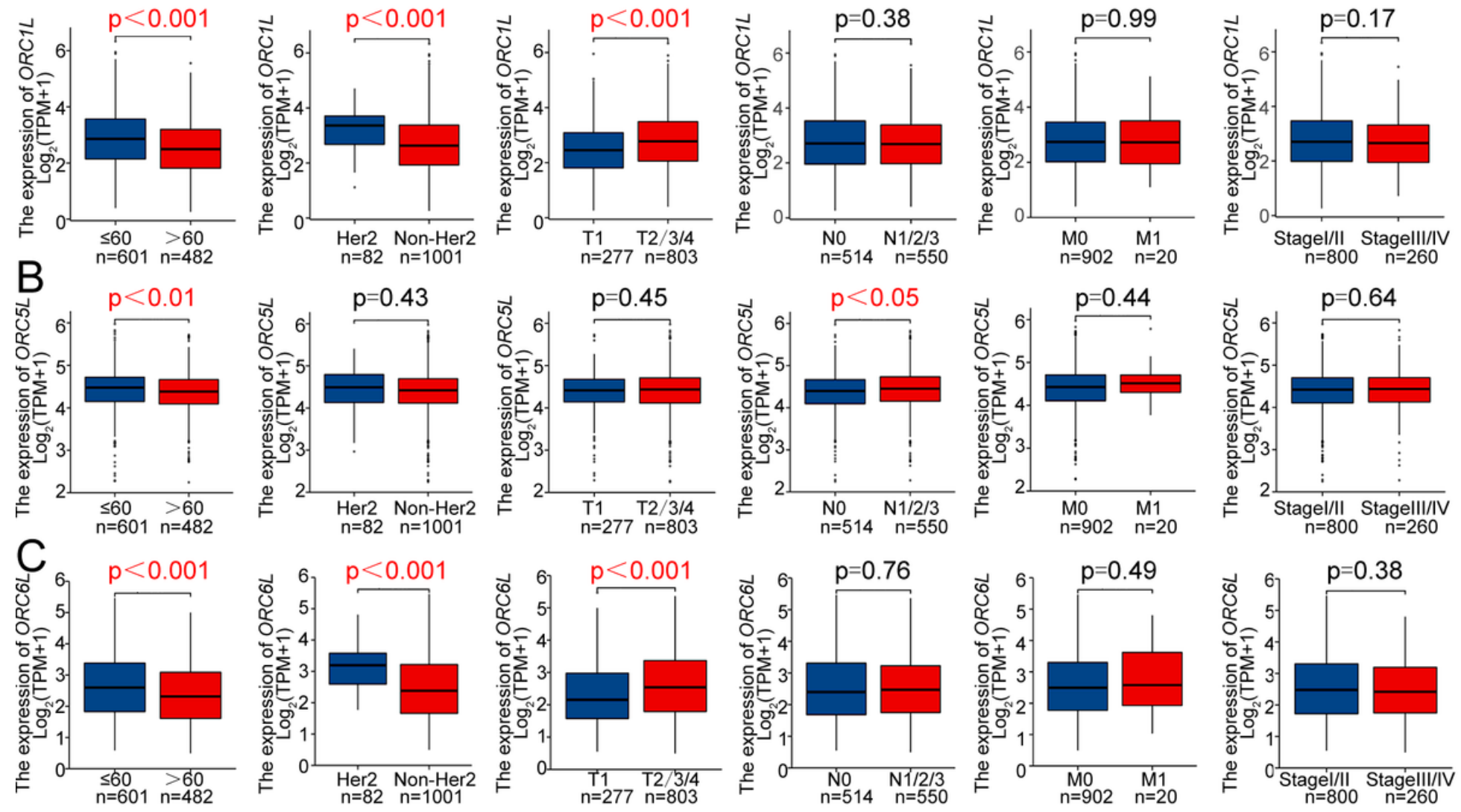

\section{Figure 3}

Relationship between the ORCs and clinicopathological features in BC (A)The relationship between ORC1L and age, molecular subtype, T stage, $\mathrm{N}$ stage, $\mathrm{M}$ stage, and pathologic stage. (B) The relationship between ORC5L and age, molecular subtype, $\mathrm{T}$ stage, $\mathrm{N}$ stage, $\mathrm{M}$ stage, and pathologic stage. (C) The relationship between ORC6L and age, molecular subtype, T stage, $\mathrm{N}$ stage, $\mathrm{M}$ stage, and pathologic stage. $n=n u m b e r$ of cases. $P$ value $<0.05$ was considered statistically significant. 

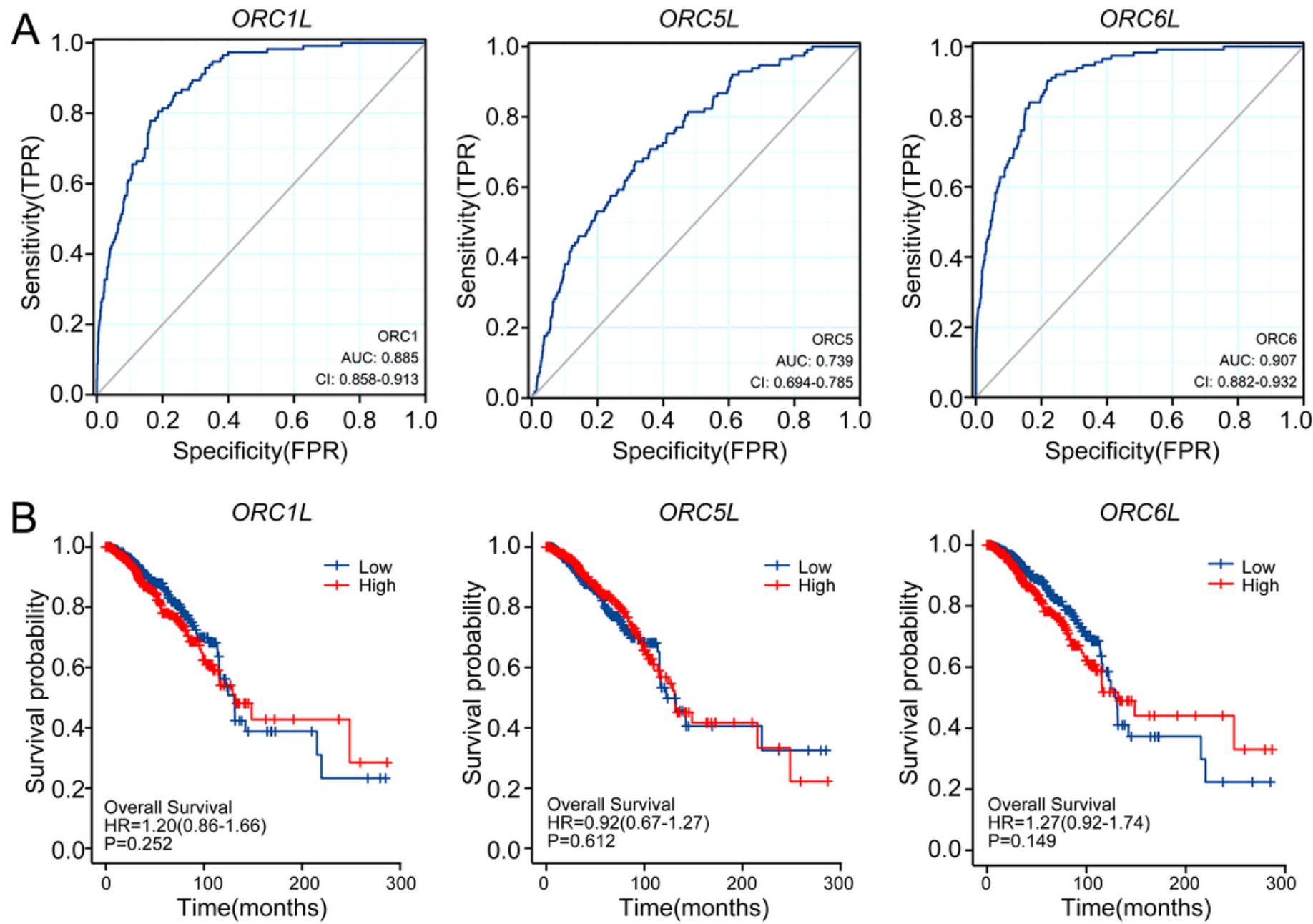

Figure 4

Diagnostic and prognostic values of ORC1L, ORC5L and ORC6L in BC (A)The ROC was plotted to estimate the diagnostic value of ORC1L $₫$ ORC5L and ORC6 expression in BC. (B)Kaplan-Meier method illustrated the impacts of ORC1L, ORC5L, and ORC6L on OS. 

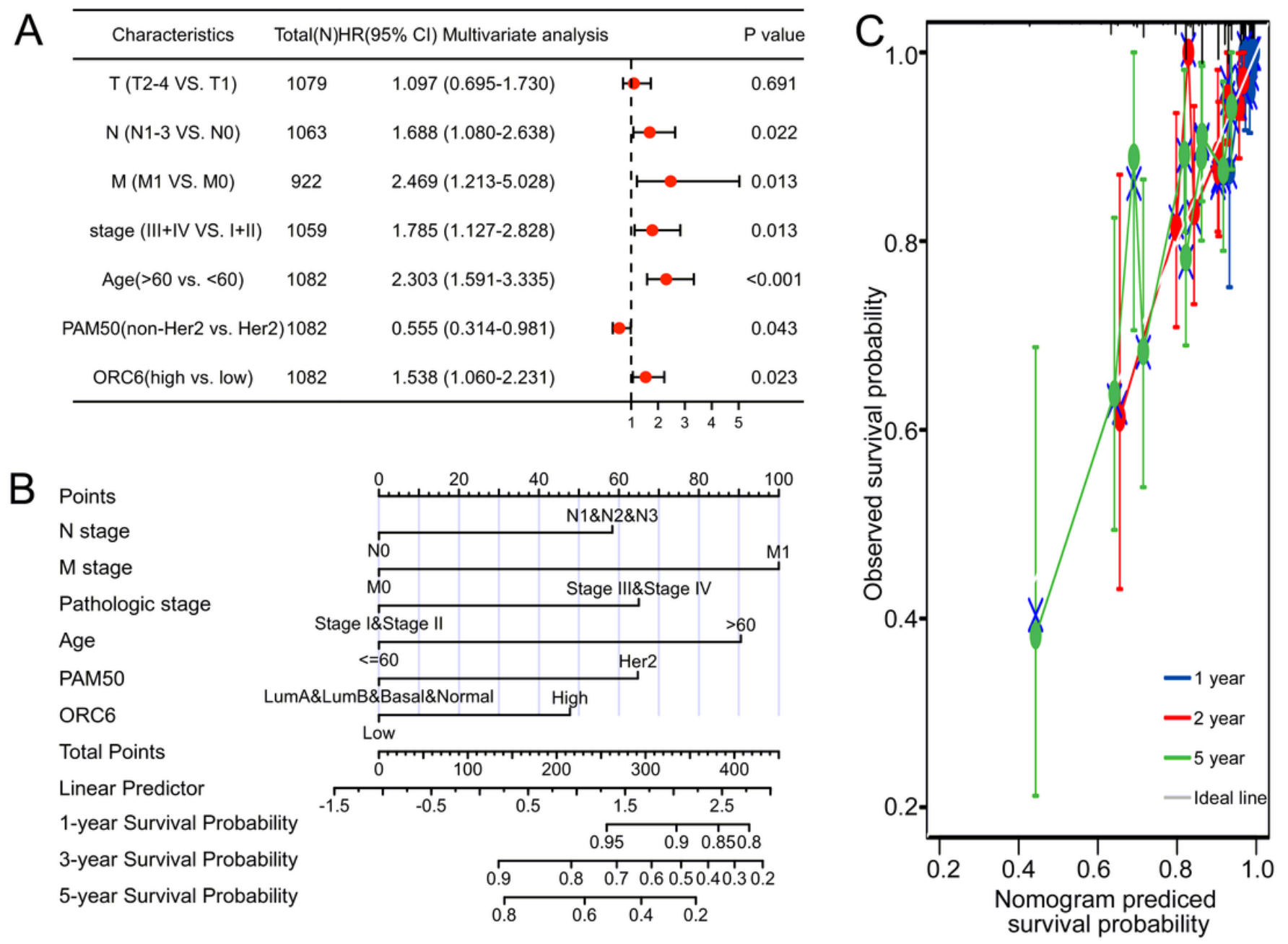

Figure 5

Multivariate Cox regression analysis and Nomogram (A) ORC6L, age, molecular subtype, T stage, $\mathrm{N}$ stage, $\mathrm{M}$ stage, and pathologic stage were included to multivariate cox regression analysis.(B)According to the nomogram, the total score was obtained after adding up the scores of each factor. The probability corresponding to the total score on the scale was the 1-year, 3-year and 5-year survival probability. (C) The calibration plot showed the mean predicted probabilities close to observed probabilities. 


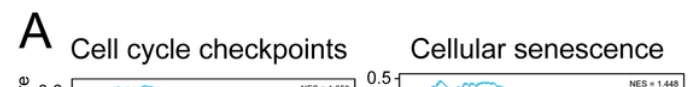

Transcriptional regulation
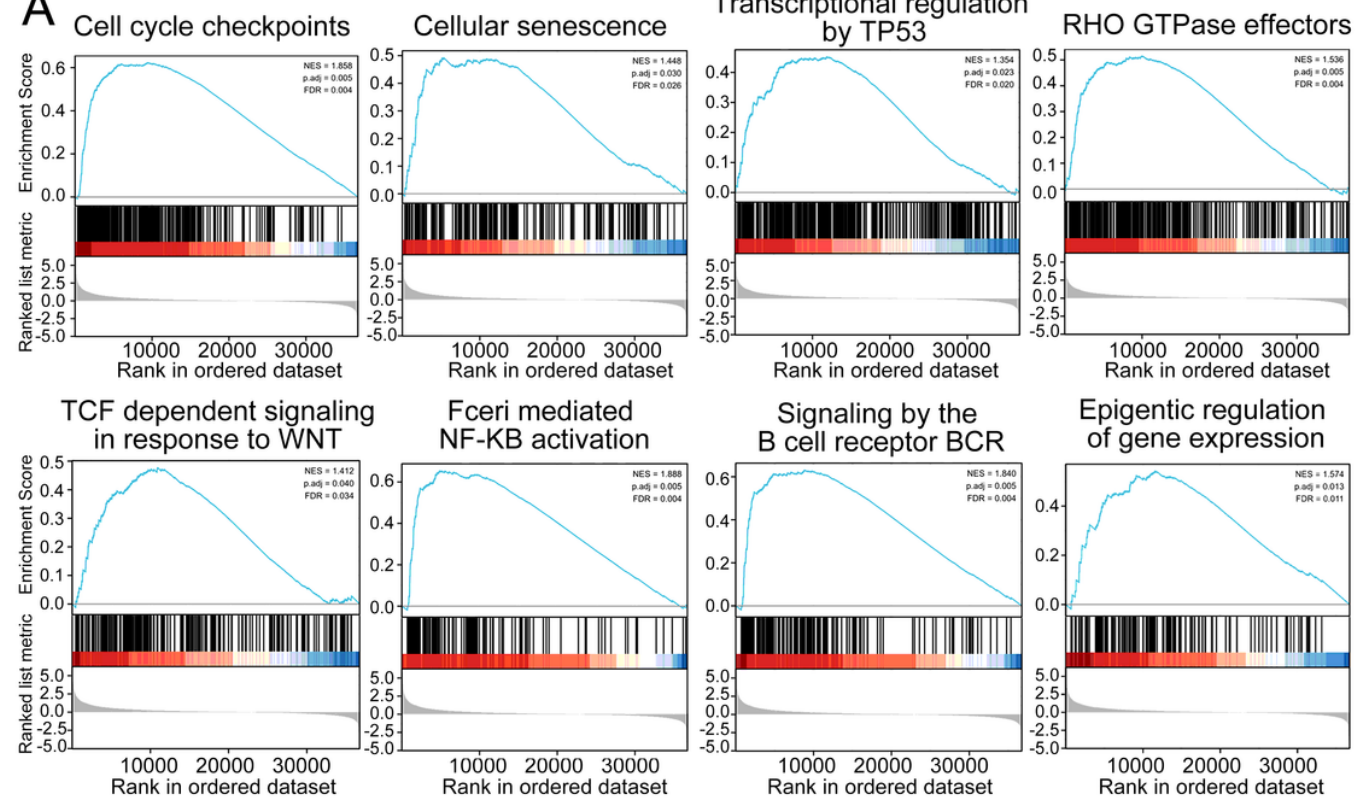

Epigentic regulation

of gene expression
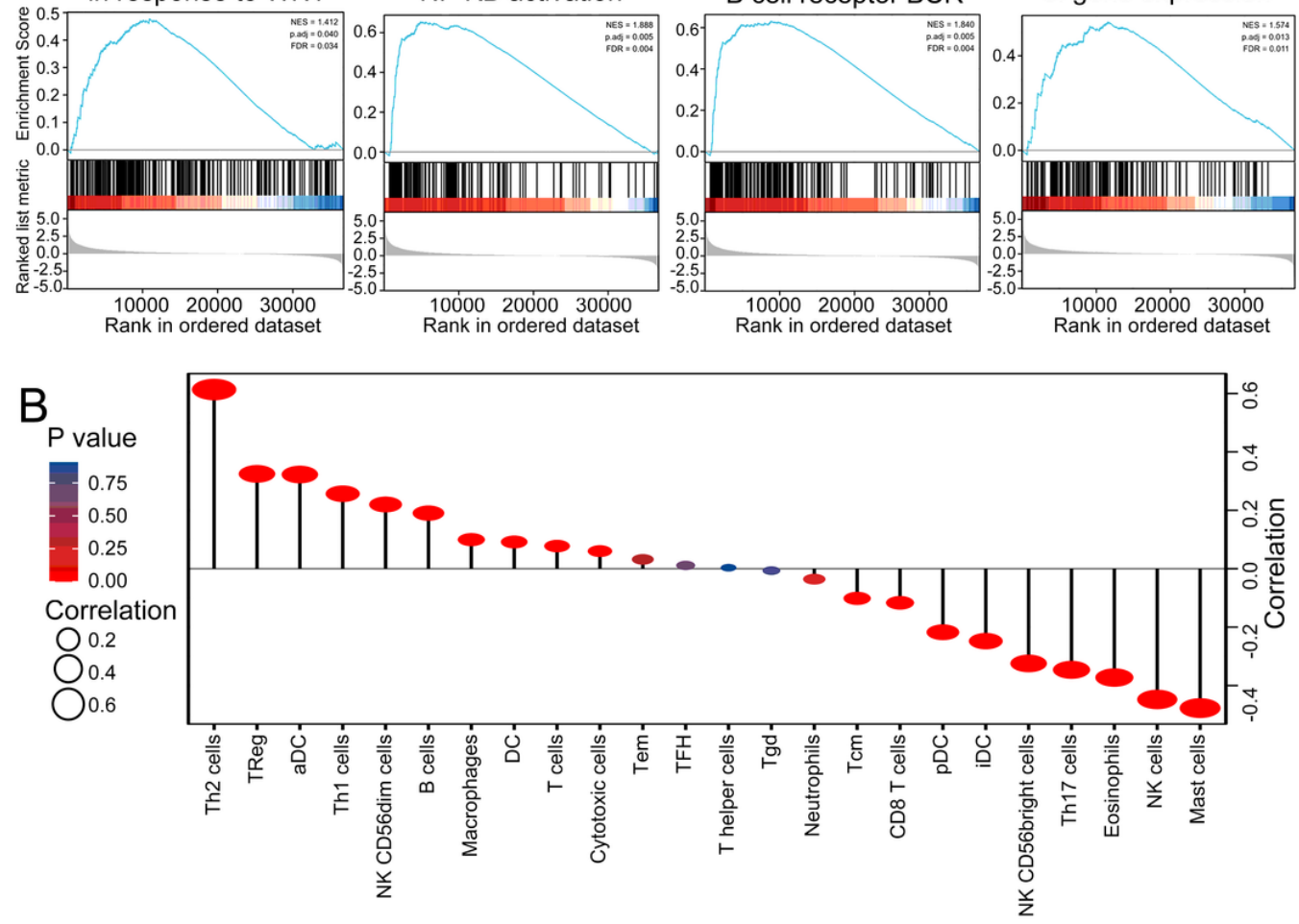

Figure 6

GSEA and Immune Cells Infiltration (A)Gene set differences between ORC6L high and ORC6L low human BC specimens revealed by GSEA. 14 biological pathways were found obviously enriched in ORC6L high BC specimens. A gene set with $\mathrm{P} \leq 0.05$ was considered to be significantly

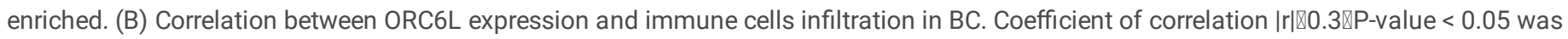
considered statistically significant. 